\title{
Generalization of Conditioned Auditory Fear is Regulated by Maternal Effects on Ventral Hippocampal Synaptic Plasticity
}

\author{
Huy-Binh Nguyen*, ${ }^{*, 6}$, Carine Parent', Yiu Chung Tse', Tak Pan Wong, ${ }^{*, 1,2,3}$ and Michael J Meaney*,1,3,4,5,7 \\ 'Douglas Mental Health University Institute, McGill University, Montréal, QC Canada; ${ }^{2}$ Department of Pharmacology and Therapeutics, McGill \\ University, Montréal, QC Canada; ${ }^{3}$ Department of Psychiatry, McGill University, Montréal, QC Canada; ${ }^{4}$ The Agency for Science, Technology and \\ Research, Singapore Institute for Clinical Sciences, Singapore, Singapore; ${ }^{5}$ Sackler Program for Epigenetics \& Psychobiology at McGill University, \\ Montréal, QC, Canada
}

\begin{abstract}
Maternal care shapes individual differences in fear-associated neural circuitry. In rats, maternal licking and grooming (LG) in early life regulates ventral hippocampal $(\mathrm{VH})$ function and plasticity in adulthood, but its consequent effect on the regulation of fear memories remains unknown. We report an effect of maternal care on generalization of learned fear, such that offspring of high LG mothers express generalized fear responses when confronted with neutral stimuli following auditory fear conditioning. These animals simultaneously display a reduction in the magnitude of VH long-term potentiation (LTP) expressed and reduced input-output transformation in Schaffer collateral synapses. Inhibition of VH-LTP during learning specifically increases fear generalization in offspring of low LG mothers during recall, suggesting a role for $\mathrm{VH}$ synaptic plasticity in the specification of fear memories. These findings suggest that rearing by low $L G$ dams enhances the efficacy of fear-related neural systems to support accurate encoding of fear memories through effects on the $\mathrm{VH}$. Neuropsychopharmacology (2018) 43, 1297-1307; doi:I0.1038/npp.2017.28I; published online 20 December 2017
\end{abstract}

\section{INTRODUCTION}

Environmental signals in early life pattern neural development in humans to produce individual differences in cognitive/emotional phenotypes. Poor quality of parental interactions in early life, such as parental neglect or abuse, alters the development of neural systems to enhance the activity of fear-associated circuitry, increasing sensitivity to potentially threatening information (Gee et al, 2013; Pollak and Kistler, 2002). Likewise, in rodents, naturally occurring variations in maternal care produce distinct cognitive and neurophysiological phenotypes in adulthood. Adult male offspring of low licking/grooming mothers (offspring of low LG mothers) learn a spatial memory task more slowly than offspring of high LG mothers (Liu et al, 2000). This is reflected in a reduction in the magnitude of long-term potentiation (LTP) in the dorsal hippocampus, a cellular

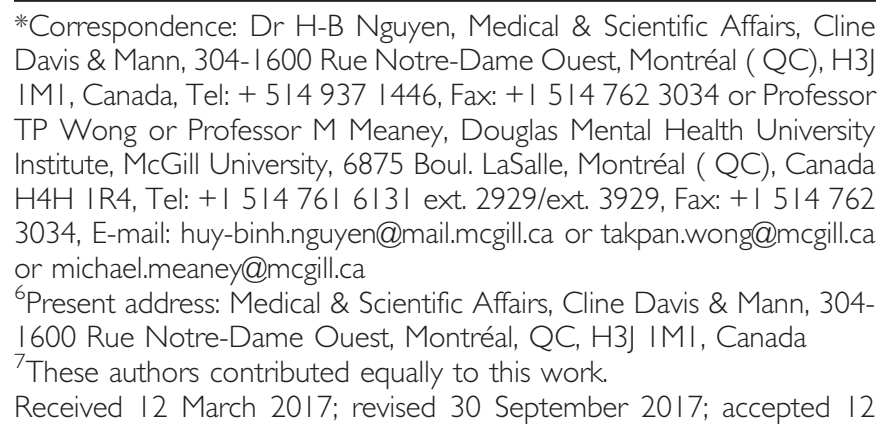
November 2017; accepted article preview online 20 November 2017 correlate of spatial learning (Bagot et al, 2009; Champagne et al, 2008; Nguyen et al, 2015). Simultaneously, the offspring of low LG mothers are more fearful and react more quickly to perceived threats (Caldji et al, 1998; Menard et al, 2004; Weaver et al, 2006), functions that are linked to the ventral hippocampus. We previously showed that offspring of low LG mothers show greater LTP and increased intrinsic neuronal excitability in the ventral hippocampus, as compared with offspring of high LG mothers, suggesting a greater capacity for handling fear-related information (Nguyen et al, 2015). Strikingly, offspring of low LG mothers display greater retention of contextual fear memories and are resistant to the impairing effects of corticosterone on synaptic plasticity and NMDA receptor function in the dorsal hippocampus (Bagot et al, 2009; 2012). Thus, rather than a global impairment in neural function, reduced maternal care in the rat may instead enhance neural function in brain regions that support fear-related learning.

Although synaptic plasticity in the amygdala alone is likely sufficient for the formation of auditory cued fear conditioning (Nabavi et al, 2014; Phillips and LeDoux, 1992; Rumpel et al, 2005), the ventral hippocampus may also contribute to the processing of fear memory. Intact ventral hippocampal $(\mathrm{VH})$ function is required for the acquisition (Maren and Holt, 2004), extinction (Sierra-Mercado et al, 2011) and renewal (Hobin et al, 2006; Jin and Maren, 2015) of conditioned cued fear, and contributes to the discrimination of conditioned vs neutral stimuli (McHugh et al, 2013; Weeden et al, 2014; Yuan et al, 2015). The ventral hippocampus is also extensively connected to regions that 
regulate fear (Bazelot et al, 2015; Hubner et al, 2014), with subpopulations of neurons contributing to the formation of anxiety (Adhikari et al, 2010; Felix-Ortiz et al, 2013), susceptibility to social defeat (Bagot et al, 2015; Calfa et al, 2007), and reward seeking (Ciocchi et al, 2015). These subpopulations are not mutually exclusive, such that many ventral CA1 neurons simultaneously project to more than one downstream site and are active during multiple tasks. These neurons may be particularly important in integrating and coordinating emotionally-salient information routing across a distributed neural network (Ciocchi et al, 2015; Jin and Maren, 2015), possibly through plastic changes in synaptic inputs from these networks in the ventral hippocampus.

As hippocampal synaptic plasticity is proposed to be a cellular correlate supporting learning, we hypothesized that programming of $\mathrm{VH}$ plasticity by maternal care results in differences in the capacity for discriminating between conditioned and neutral cues during fear learning. Compared with offspring of high LG mothers, we find that offspring of low LG mothers freeze significantly less in response to a neutral tone than to the conditioned tone following cued fear conditioning. Generalized freezing in offspring of high LG mothers could result from reduced $\mathrm{VH}$ LTP expression in these rats when comparing to offspring of low LG mothers. We found that selective blockade of $\mathrm{VH}$ LTP increases generalized freezing in offspring of low but not of high LG mothers.

\section{MATERIALS AND METHODS}

\section{Characterization of Maternal Behavior}

Maternal behavior was characterized as previously described (Champagne et al, 2003). The maternal behavior of LongEvans rat dams (Charles River, St. Constant, QC, Canada) was scored every 3 mins for five 75 -min observation periods each day during the first 6 postnatal days to score the frequency of licking and grooming (LG), generating 125 observations per animal. Scores within a cohort were tested for normality using the Kolmogorov-Smirnov test. Dams for which the mean LG frequency lay 1 SD above or below the cohort mean were designated as high and low LG mothers, respectively. Animals were weaned at postnatal day 21, pairhoused with littermates and maintained with minimal handling until being killed for experiments in adulthood ( postnatal day 120). Food and water were available ad libitum. For studies in uncharacterized animals used in experiments where no direct comparison between offspring of high and low LG dams was made, adult male rats were obtained (Charles River) at postnatal day 100 and kept onlocation for at least 2 weeks to allow habituation to the new facility before being killed. All procedures conformed to the guidelines of the Canadian Council on Animal Care with protocols approved by the Facility Animal Care Committee at Douglas Mental Health University Institute. Experiments were conducted by an experimenter blind to animal group.

\section{Slice Preparation}

Acute slices were prepared as previously described (Nguyen et al, 2015). In brief, animals were anesthetized immediately following removal from the home cage by inhalation of isoflurane and rapidly decapitated. Brains were removed and cut into slices using a vibratome (Leica, Concord, ON, Canada) in slicing solution containing (in $\mathrm{mM}$ ): 252 sucrose, $2.5 \mathrm{KCl}, 4 \mathrm{MgCl}_{2}, 0.1 \mathrm{CaCl}_{2}, 1.25 \mathrm{KH}_{2} \mathrm{PO}_{4}, 26 \mathrm{NaHCO}_{3}$ and 10 glucose $(\sim 360 \mathrm{mOsmol} / \mathrm{l})$. Slices were incubated at $32{ }^{\circ} \mathrm{C}$ for $1 \mathrm{~h}$ in carbogenated (95\% oxygen, 5\% $\mathrm{CO}_{2}$ ) artificial cerebrospinal fluid (aCSF) containing (in $\mathrm{mM}$ ): $125 \mathrm{NaCl}$, $2.5 \mathrm{KCl}, 1 \mathrm{MgCl} 2,2 \mathrm{CaCl} 2,1.25 \mathrm{NaH} 2 \mathrm{PO} 4,26 \mathrm{NaHCO} 3$, and 25 glucose $(\sim 310 \mathrm{mOsmol} / \mathrm{l})$. Slices were allowed to recover at room temperature for $1 \mathrm{~h}$ before being transferred to a recording chamber for the start of recordings.

\section{Electrophysiology}

Field excitatory postsynaptic potentials (fEPSPs) were evoked using bipolar tungsten stimulating electrodes placed in the stratum radiatum of area CA1, and responses were detected by aCSF-filled glass recording electrodes. Field recordings were performed in the presence of $5 \mu \mathrm{M}$ bicuculline methobromide (Tocris, Bristol, UK) to block $\gamma$ aminobutyric acid type $\mathrm{A}\left(\mathrm{GABA}_{\mathrm{A}}\right)$ receptor-mediated inhibitory postsynaptic transmission. Input-output relationships at Schaffer collateral synapses were constructed by recording fEPSPs in response to presynaptic fiber volleys of amplitudes increasing stepwise in $0.1 \mathrm{mV}$ increments between 0.1 and $1.0 \mathrm{mV}$. fEPSPs were evoked at $0.2 \mathrm{~Hz}$ and the average of 10 traces was used to calculate fEPSP slope and peak at each input level. The formation of LTP was examined by recording baseline fEPSPs for $20 \mathrm{~min}$ before LTP induction. LTP induction was achieved by tetanic stimulation (three trains of 100 pulses delivered at $100 \mathrm{~Hz}$, separated by $5 \mathrm{~min}$ inter-train intervals). fEPSPs were evoked at $0.05 \mathrm{~Hz}$ and the average of six traces was used to calculate fEPSP slope every two minutes. Traces were excluded if baseline responses were not stable ( $> \pm 10 \%$ change).

Whole-cell patch clamp recordings of evoked excitatory postsynaptic currents (eEPSCs) were performed on ventral hippocampus CA1 neurons in voltage-clamp mode with pipettes containing (in $\mathrm{mM}$ ): 110 Cs-gluconate, 17.5 CsCl, 10 HEPES, $2 \mathrm{MgCl}_{2}, 0.5$ EGTA, 4 ATP, 5 QX-314, and 0.03 Tat-GluA1ct peptide (YGRKKRRQRRR-830QSINEAIRTSTLPRNSG846) or control unrelated TatControl peptide (YGRKKRRQRRR-VYKYGGYNE) (Lyophilized powder, soluble in water, purity at $\geqslant 95 \%$ ) (GenScript, Piscataway, NJ, USA) with $\mathrm{pH}$ adjusted to 7.2 with $\mathrm{CsOH}$ ( $290 \mathrm{mOsm}$ ) (Yu et al, 2008). Neurons were held at $-60 \mathrm{mV}$, and EPSCs were evoked from Schaffer collaterals by a bipolar tungsten electrode placed in the stratum radiatum at a rate of $0.05 \mathrm{~Hz}$ and the average of three traces was used to calculate the fEPSP slope at each minute. Recordings were performed in the presence of $5 \mu \mathrm{M}$ bicuculline (Tocris Bioscience). Initial EPSC amplitudes were normalized to $\sim 100 \mathrm{pA}$, and 5 min baseline periods were acquired before LTP was induced. Traces were excluded if baseline responses were not stable ( $> \pm 10 \%$ change). LTP was induced within $10 \mathrm{~min}$ or less of cell break-in to avoid wash-out of plasticity-related proteins. LTP was induced by stimulating Schaffer collaterals at a rate of $2 \mathrm{~Hz}$ for $100 \mathrm{~s}$, while cells were voltage-clamped at $-5 \mathrm{mV}$. Access resistance was continuously monitored during recordings, and 
recordings were excluded if access resistance exceeded $25 \mathrm{M} \Omega$ or changed by $>5 \mathrm{M} \Omega$ during the course of recording.

Recordings were amplified by Multiclamp 700B (Axon), low-pass filtered at $1 \mathrm{kHz}$, and sampled at $10 \mathrm{kHz}$. Recordings were digitized by Digidata 1400 (Axon), and stored in a PC for later offline analysis. All data were analyzed using Clampfit (Molecular Devices, Sunnyvale, CA, USA). Recordings were made at room temperature $\left(\sim 22^{\circ} \mathrm{C}\right)$.

\section{Fear Conditioning}

Conditioning and testing of retention $24 \mathrm{~h}$ later was performed in fear conditioning chambers (Med Associates). The chamber was modified between conditioning and testing days in order to minimize contextual cues. During habituation and retention testing, chambers were fitted with a smooth stainless steel pan filled with corn cob bedding to serve as the chamber floor. During conditioning, visual, tactile, and olfactory cues presented in the context were changed. Visual cues in the room were changed. Tactile cues were changed by fitting chambers with a grid shock floor made of parallel stainless steel bars. Distinct odor cues were presented by placing 2-3 drops of 30\% acetophenone diluted in propylene glycol in a petri dish under the testing chamber such that animals could smell but not touch the odorant.

Animals were habituated to manipulations associated with testing and pre-exposed to the retention chamber for $5 \mathrm{~min}$ per day for 2 days prior to conditioning. For auditory fear conditioning, animals were placed into fear conditioning chambers. Following a $5 \mathrm{~min}$ habituation period, animals were presented with two conditioned stimulus/unconditioned stimulus ( $\mathrm{CS}^{+} / \mathrm{US}$ ) couplets separated by $60 \mathrm{~s}$. $\mathrm{CS}^{+} / \mathrm{US}$ couplets consisted of $10 \mathrm{~s}$ presentation of a $2000 \mathrm{~Hz}, 70 \mathrm{~dB}$ pure tone that coterminates with a $1 \mathrm{~s}, 0.5 \mathrm{~mA}$ footshock. Testing of retention occurred $24 \mathrm{~h}$ later. Animals were placed into testing chambers. Following a 5 min context habituation period, animals were presented with a novel, non-conditioned, neutral stimulus ( $\mathrm{CS}^{-}, 500 \mathrm{~Hz}, 70 \mathrm{~dB}$ pure tone) for $2 \mathrm{~min}$. After a $5 \mathrm{~min}$ inter-stimulus period, animals were presented with the conditioned stimulus $\left(\mathrm{CS}^{+}, 2000 \mathrm{~Hz}\right.$, $70 \mathrm{~dB}$ pure tone) for $2 \mathrm{~min}$. Freezing behavior was defined as the absence of all movement except those associated with respiration and scored during retention testing for the duration of the context habituation, inter-stimulus, and stimulus presentation periods as previously described (Bagot et $a l, 2009)$ by an experimenter blind to experimental groups.

\section{Surgery and Infusions}

Guide cannulae (26 gauge, $11 \mathrm{~mm}$, stainless steel) were bilaterally implanted directed at coordinates (in $\mathrm{mm}$ ): $\mathrm{AP}$ -5.2 posterior, $\mathrm{ML} \pm 5.0$ lateral to Bregma, $\mathrm{DV}-6.0$ ventral to dura. Guide cannulae were fixed to the skull with three anchoring screws and dental cement. Stainless steel stylets were inserted to prevent occlusion of the cannulae, and were removed only for infusions. Following surgery, animals were allowed to recover for at least 10 days before testing. For testing, animals were habituated to manipulations associated with infusions and testing, as well as to the retention chamber as described above. After 2 days of habituation, infusion cannulae (36 gauge, $12 \mathrm{~mm}$, stainless steel, $1.6 \mathrm{~mm}$ protrusion from guide) were inserted into guide cannulae to achieve a final target location of $7.6 \mathrm{~mm}$ ventral to the dura. Tat-GluA1ct peptide and Tat-Control peptide were suspended in $0.1 \mathrm{M}$ phosphate-buffered saline at a concentration of $30 \mu \mathrm{M} .10 \mu \mathrm{l}$ Hamilton syringes attached to infusion cannulae by flexible tubing were used to bilaterally infuse $1 \mu \mathrm{l}$ of peptide solution over $60 \mathrm{~s}$, with an expected spread of up to $2 \mathrm{~mm}$ away from the injection site (Peterson, 1998). Internal cannulae were left in place for an additional $60 \mathrm{~s}$ to allow for bolus absorption. Blocking stylets were then re-inserted into guide cannulae. After $15 \mathrm{~min}$, animals were placed into testing chambers for conditioning as described above.

\section{Histology}

After completion of behavioral experiments, rats were anesthetized by inhalation of isoflurane and killed by decapitation. Brains were rapidly removed and post-fixed in $10 \%$ formalin overnight, after which they were transferred to $20 \%$ sucrose/formalin solution. Brains were subsequently sliced into $40 \mu \mathrm{m}$-thick coronal sections on a cryostat and mounted on gelatin-coated glass slides. Sections were then stained with cresyl violet and dehydrated through increasing alcohol concentrations, cleared with Neoclear and coverslipped with Permount. Slides were visualized on a microscope to verify track marks formed by cannulae tips.

\section{Reagents}

All reagents were obtained from Sigma-Aldrich (St. Louis, MO, USA) unless otherwise specified.

\section{Statistical Analysis}

Statistical analyses were performed using SPSS 20.0 (IBM, Armonk, NY, USA). Graphs were plotted using Graphpad Prism 4. Data were analyzed using two-way ANOVA (with repeated measures when dependent variables were measured at multiple within-subject levels), and significant interactions were decomposed using simple main effects and Bonferronicorrected pairwise comparisons.

\section{RESULTS}

We began by studying the effect of maternal care on $\mathrm{VH}$ function. We assessed synaptic transmission with studies of input-output transformation at Schaffer collateral synapses by stimulating presynaptic fiber volleys and recording the postsynaptic fEPSPs elicited by presynaptic firing. The relation between the output levels measured by the slope of the fEPSP was plotted as a function of the input level elicited, as measured by the fiber volley amplitude. Maternal care exerted a striking effect such that output fEPSPs elicited in offspring of low LG mothers were significantly greater than those elicited in offspring of high LG mothers in response to presynaptic fiber volleys of equivalent amplitude (Figure 1a; slope: repeated measures ANOVA, fiber volley $\times$ maternal care interaction, $\mathrm{F}_{(9,99)}=3.406, P=0.001$; amplitude: repeated measures ANOVA, effect of maternal care, $\mathrm{F}_{(1,11)}=4.998, P=0.047 ; n=7$ slices from four rats/group). We next examined the formation of LTP in the ventral 

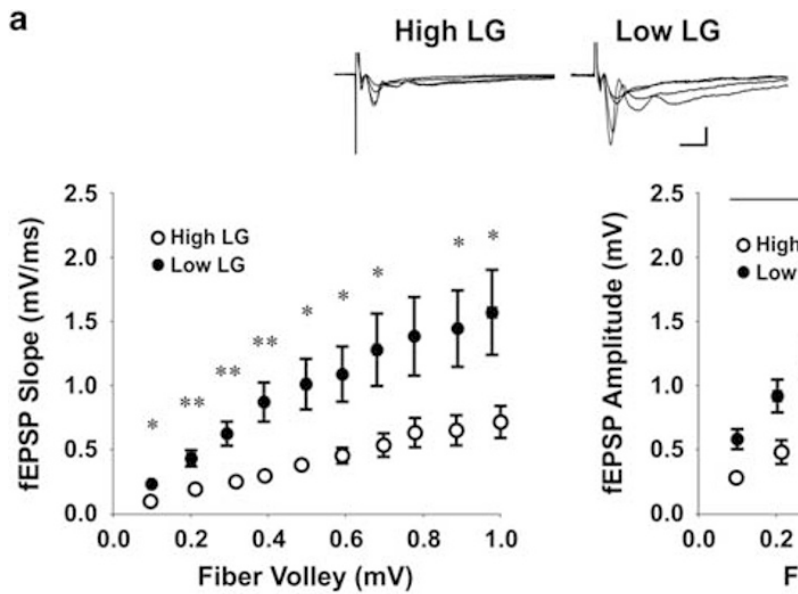

b

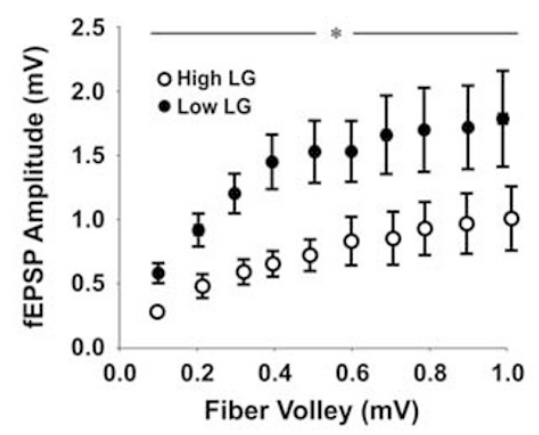

High LG
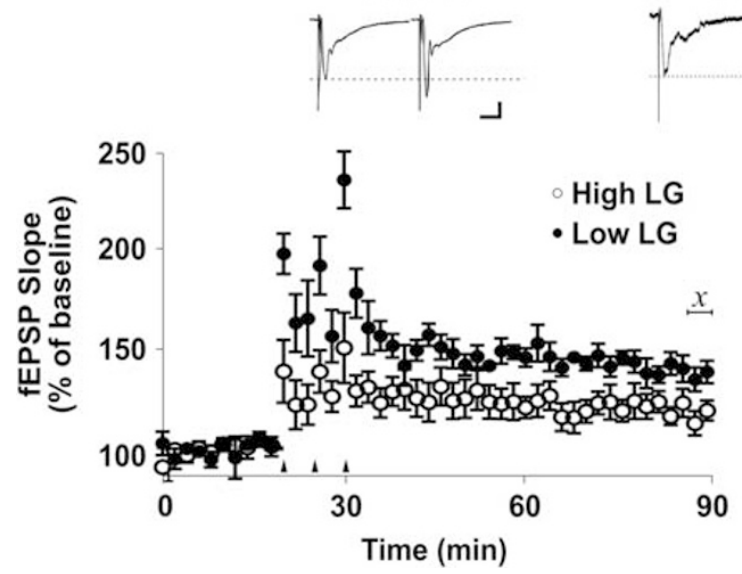

\section{Low LG}
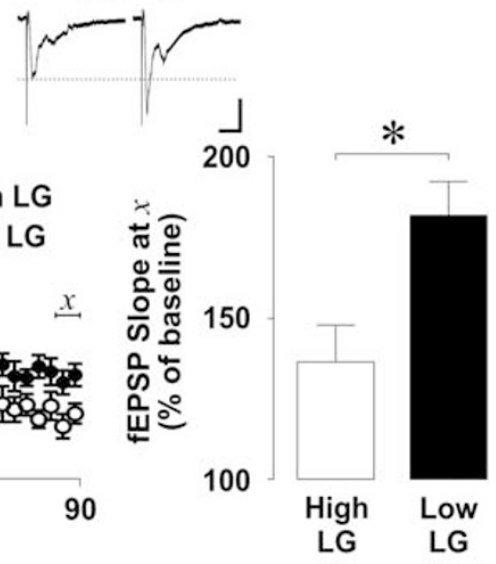

Figure I Regulation of ventral hippocampal synaptic input-output transformation and plasticity by maternal care. (a) Synaptic input-output transformation at Schaffer collateral synapses of the ventral hippocampus. Top: representative traces of field excitatory postsynaptic potentials (fEPSPs) elicited by presynaptic fiber volleys of increasing intensity. Scale bars represent $0.5 \mathrm{mV}$ (vertical) and $10 \mathrm{~ms}$ (horizontal). Bottom left: scatter plot of mean \pm SEM slope of evoked output fEPSPs elicited in response to increasing strength of fiber volley input. Bottom right: scatter plot of mean \pm SEM amplitude of evoked output fEPSPs elicited in response to increasing strength of fiber volley input. Output fEPSPs elicited from ventral hippocampal slices of offspring of low LG mothers were significantly greater than those elicited from offspring of high LG mothers in response to equivalent presynaptic fiber volley input. ( $n=7$ slices from four rats/ group) $* P<0.05$, ** $P<0.0$ I. (b) Long-term potentiation (LTP) at Schaffer collateral synapses of the ventral hippocampus. Top: representative traces of field excitatory postsynaptic potentials (EEPSPs) before and I h after tetanic stimulation. Dashed line indicates baseline fEPSP amplitude. Scale bars represent $0.25 \mathrm{mV}$ (vertical) and $20 \mathrm{~ms}$ (horizontal). Bottom left: scatter plot of mean \pm SEM slope of evoked fEPSPs before and I h after LTP induction. Arrows indicate tetanic stimulation $(3 \times 100 \mathrm{~Hz}, 100$ pulses). Bottom right: mean+SEM of fEPSP slope normalized to baseline I h after tetanus. The slope of fEPSPs recorded from ventral hippocampal slices of offspring of low LG mothers displayed significantly greater potentiation I h after tetanus delivery when compared with fEPSPs recorded from offspring of high LG mothers. Statistical analysis was performed on the average of values underlying $\chi$ reflecting LTP at I $\mathrm{h}$ following tetanic stimulation. (offspring of high LG dams: $n=6$ slices from four rats; offspring of low LG dams: $n=5$ slices from four rats) $* P<0.05$.

hippocampus. As previously reported (Nguyen et al, 2015), offspring of low LG mothers displayed greater VH-LTP than did offspring of high LG mothers (Bonferroni-corrected pairwise comparison, $P=0.016) 60 \mathrm{~min}$ after tetanic stimulation (Figure $1 \mathrm{~b}$; period $\times$ maternal care interaction, $\mathrm{F}_{(1,9)}=5.263 ; P=0.04 ;$ offspring of high LG dams: $n=5$ slices from four rats; offspring of low LG dams: $n=6$ slices from four rats).

The formation of synaptic plasticity, including LTP, is a cellular correlate of learning and memory formation. To study whether the changes in VH synaptic plasticity associate with differences in learning and memory, we examined fearassociated freezing behavior in animals conditioned to auditory tone-shock pairings. During conditioning, unconditioned orienting (repeated measures ANOVA, main effect of maternal care, $\left.\mathrm{F}_{(1,17)}=1.873, \quad P=0.19\right)$ and locomotor (repeated measures ANOVA, main effect of maternal care, $\left.\mathrm{F}_{(1,17)}=0.91, P=0.35\right)$ responses to auditory and footshock stimuli were not significantly different between offspring of high and low LG dams (Supplementary Figure S1). During retention, both offspring of high and low LG mothers displayed significantly greater freezing in response to the conditioned shock-associated $\left(\mathrm{CS}^{+}\right)$tone than to the background context (Bonferroni-corrected pairwise comparisons, $P=8.04 \times 10^{-5}$ and $P=0.001$, respectively), but did not differ in freezing from each other (Bonferroni-corrected pairwise comparison, $P=0.533)$. However, when presented with a neutral, unconditioned stimulus $\left(\mathrm{CS}^{-}\right)$, only offspring of high LG mothers froze significantly (Bonferroni-corrected pairwise comparison, $P=1.1 \times 10^{-5}$ ) compared with 


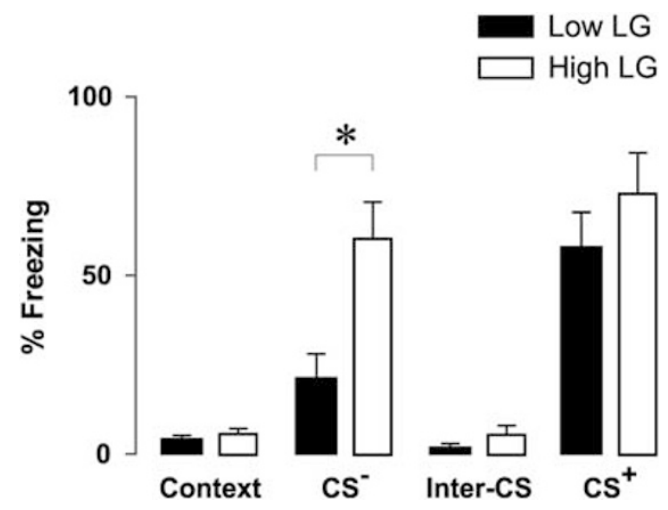

Figure 2 Variations in early life maternal care regulate generalization of fear-associated freezing following auditory fear conditioning. Fear-associated freezing following cued fear conditioning in response to a neutral stimulus $\left(\mathrm{CS}^{-}\right)$and the conditioned stimulus $\left(\mathrm{CS}^{+}\right)$. Bar graphs denote mean+SEM of percent of time during each stimulus presentation period spent freezing. Offspring of high LG mothers froze significantly more than offspring of low LG mothers when presented with $\mathrm{CS}^{-}$, and only offspring of high LG mothers froze significantly more in response to $\mathrm{CS}^{-}$than to the background context. (offspring of high LG dams: $n=12$ rats; offspring of low $L G$ dams: $n=1 \mid$ rats $) * P<0.05$.

background, and froze significantly more than offspring of low LG mothers (Bonferroni-corrected pairwise comparison, $P=0.002$ ) (Figure 2; repeated measures ANOVA, maternal care $\times$ stimulus interaction, $\mathrm{F}_{(3,63)}=4.343 ; P=0.008$; offspring of low LG dams: $n=11$ rats, offspring of high LG dams: $n=12$ rats ). Conversely, offspring of low LG mothers did not display significant freezing in response to the neutral stimulus compared with background (Bonferroni-corrected pairwise comparison, $P=0.412$ ). We conclude that offspring of high but not of low LG mothers generalize conditioned fear responses to a neutral stimulus.

LTP formation is mediated by exocytosis of AMPA receptors to postsynaptic membranes. Following induction of LTP, intracellular signaling cascades result in the phosphorylation of serine residues 831 and 845 on the C-terminal of the GluA1 AMPA receptor subunit, which signals their delivery to postsynaptic membranes (Rumpel et al, 2005; Shi et al, 2001; Yu et al, 2008). To selectively target and block LTP in the ventral hippocampus, we adopted an interfering peptide approach to block GluA1containing AMPA receptor insertion ( $\mathrm{Yu}$ et al, 2008). We confirmed that baseline synaptic transmission was not affected by the presence of Tat-GluAlct by recording evoked EPSCs in VH CA1 neurons (Supplementary Figure S2; paired samples Student's $t$-test, $\left.\mathrm{t}_{(3)}=0.635, P=0.57\right)$. We validated the Tat-GluAlct blockade of LTP formation in VH neurons by recording LTP of evoked EPSCs in CA1 pyramidal neurons of the ventral hippocampus in uncharacterized animals in the presence of Tat-GluA1ct or a control Tat-Ctrl peptide. LTP recorded in the presence of TatGluA1ct was significantly (Bonferroni-corrected pairwise comparison, $P=0.006$ ) reduced in magnitude compared with that recorded in the presence of Tat-Ctrl. EPSCs recorded in the presence of Tat-GluAlct did not undergo significant potentiation when compared with baseline (Bonferronicorrected pairwise comparison, $P=0.63$ ). We conclude that Tat-GluA1ct inhibits LTP formation in VH CA1 pyramidal neurons (Figure 3; repeated measures ANOVA: period $x$ peptide interaction, $\mathrm{F}_{(1,8)}=13.646, P=0.006$; Tat-Ctrl: $n=5$ cells from five rats; Tat-GluA1ct: $n=5$ cells from five rats).

Although synaptic plasticity in the amygdala is thought to underlie the formation of the association between the auditory $\mathrm{CS}^{+}$and the unconditioned stimulus (Nabavi et al, 2014; Phillips and LeDoux, 1992; Rumpel et al, 2005), discrimination between predictive and neutral cues involves extra-amygdalar regions such as the ventral hippocampus and prefrontal cortex (Likhtik et al, 2014; McHugh et al, 2013). We hypothesized that LTP in the ventral hippocampus during learning may contribute to stimulus discrimination during recall. To specifically block LTP formation in the ventral hippocampus, we infused Tat-GluAlct or Tat-Ctrl peptide into the ventral hippocampus of offspring of high and low LG mothers, $15 \mathrm{~min}$ before conditioning, and examined freezing during presentation of $\mathrm{CS}^{-}$followed by $\mathrm{CS}^{+}$stimuli $24 \mathrm{~h}$ later, as previously. Unconditioned orienting (repeated measures ANOVA, main effect of maternal care, $\mathrm{F}_{(1,23)}=0.88, P=0.36$ ) and locomotor (repeated measures ANOVA, main effect of maternal care, $\mathrm{F}_{(1,23)}=0.429$, $P=0.52$ ) responses to auditory and footshock stimuli were not significantly different between Tat-GluA1ct- or Tat-Ctrlinfused offspring of high and low LG dams during conditioning (Supplementary Figure S3). During retention, we found a significant three-way interaction between stimulus, peptide, and maternal care (Figure 4; $\mathrm{F}_{(3,66)}=4.905, P=0.004$; Tat-Ctrl-infused offspring of low LG dams: $n=9$ rats; Tat-GluAlct-infused offspring of low LG dams: $n=7$ rats; Tat-Ctrl-infused offspring of high LG dams: $n=5$ rats; Tat-GluAlct-infused offspring of high LG dams: $n=5$ rats). Decomposition of this interaction revealed a significant stimulus $\times$ peptide interaction in offspring of low LG mothers $\left(\mathrm{F}_{(3,42)}=4.164, P=0.011\right)$, but not in offspring of high $\mathrm{LG}$ mothers $\left(\mathrm{F}_{(3,24)}=0.415, P=0.744\right)$, such that offspring of low LG mothers infused with TatGluA1ct froze significantly (Bonferroni-corrected pairwise comparison, $P=0.007)$ more in response to the $\mathrm{CS}^{-}$ presentation than Tat-Control infused animals. In contrast, there was no significant effect of peptide on freezing in response to the background context (Bonferroni-corrected pairwise comparison, $P=0.85$ ) or to the conditioned stimulus (Bonferroni-corrected pairwise comparison, $P=0.445)$. We conclude that occlusion of LTP formation in the ventral hippocampus during learning reduces discrimination between neutral and conditioned cues in offspring of low LG mothers during retention.

\section{DISCUSSION}

We present evidence for an effect of early life maternal care on VH synaptic function and plasticity, which may influence generalization of conditioned fear. Offspring of high LG mothers, compared with offspring reared by low LG mothers, showed reduced synaptic transmission and magnitude of LTP expressed in the ventral hippocampus, which associates with significantly greater freezing in response to a neutral cue after auditory fear learning. Specific occlusion of LTP-associated postsynaptic AMPA receptor insertion in the ventral hippocampus during learning, which is increased in offspring of low LG mothers, increases fear-associated 


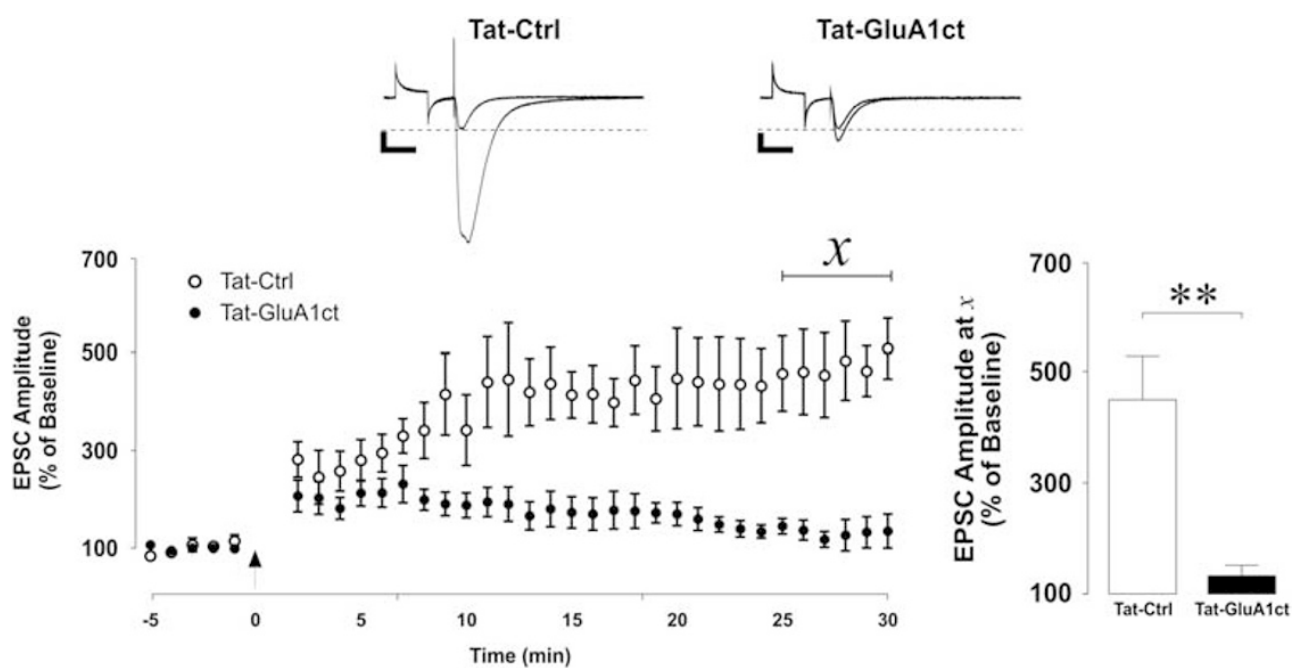

Figure 3 Synthetic AMPA receptor C-terminal tail decoy peptide inhibits LTP formation in ventral hippocampal CAI neurons. Long-term potentiation (LTP) of excitatory postsynaptic currents (EPSCs) in the presence of an AMPA receptor C-terminal tail mimic (Tat-GluAIct) or control (Tat-Ctrl) peptide. Top: representative traces of evoked EPSCs before and 30 mins after LTP induction. Transient response to square-wave command voltage step to monitor input resistance precedes the electrode-elicited AMPA receptor-mediated EPSC. Scale bars represent 100 pA (vertical) and 50 ms (horizontal). Bottom left: scatter plot of mean \pm SEM amplitude of evoked EPSCs before and 30 mins after LTP induction. Arrow indicates LTP induction (2 Hz, 50 pulses delivered while cell was voltage-clamped at $-5 \mathrm{mV}$ ). Bottom right: mean+SEM of EPSC amplitude normalized to baseline $30 \mathrm{~min}$ after LTP induction. LTP of EPSCs was blocked when recorded in the presence of Tat-GluA I ct. Statistical analysis was performed on the average of values underlying $\chi$ reflecting LTP. (Tat-Ctrl: $n=5$ cells from five rats; Tat-GluAI ct: $n=5$ cells from five rats) $* * P<0.01$.
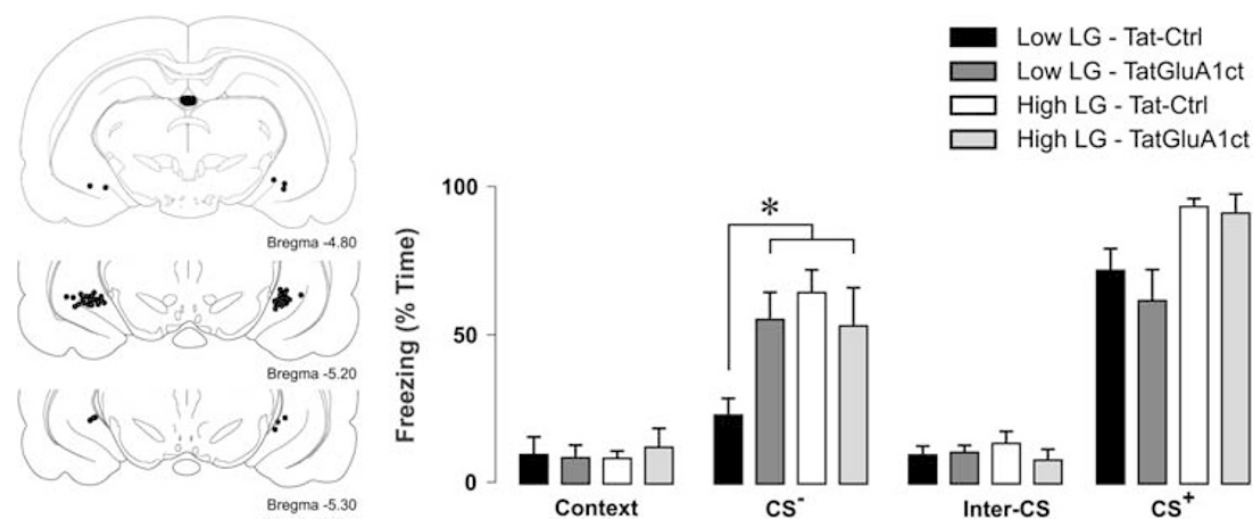

Figure 4 Inhibition of ventral hippocampal LTP increases generalization of conditioned fear in offspring of low LG dams. Left: reconstruction of coronal cross-section depicting the locations of verified cannulae tip placements in the ventral hippocampus. Right: effect of ventral hippocampal infusion of TatGluA Ict on fear-associated freezing following cued fear conditioning in response to a neutral stimulus $\left(\mathrm{CS}^{-}\right)$and the conditioned stimulus $\left(\mathrm{CS}^{+}\right)$. Bar graphs denote mean+SEM of percent of time during each stimulus presentation period spent freezing. Offspring of low LG mothers infused with Tat-GluA I ct in their ventral hippocampus froze significantly more in response to $\mathrm{CS}^{-}$than offspring of low LG mothers infused with Tat-Ctrl when presented with CS ${ }^{-}$. (Tat-Ctrlinfused offspring of low LG dams: $n=9$ rats; Tat-GluAl ct-infused offspring of low LG dams: $n=7$ rats; Tat-Ctrl-infused offspring of high $L G$ dams: $n=5$ rats; Tat-GluAl ct-infused offspring of high LG dams: $n=5$ rats) $* P<0.05$.

freezing in response to the neutral cue in offspring of low LG mothers during memory recall. Our findings support a role for learning-associated synaptic plasticity in the ventral hippocampus in the discrimination of conditioned $v s$ neutral cues during recall, and suggest that this capacity for cue discrimination in adulthood is elaborated in association with low LG rearing in early life.

The hippocampus is heterogeneous along its longitudinal axis: the ventral hippocampus is distinct from the dorsal subregion in its physiological properties (ie, excitability, plasticity) (Bittner et al, 2012; Dougherty et al, 2012; Maggio and Segal, 2007) as well as its extrinsic anatomical connectivity (Risold and Swanson, 1996; Swanson and Cowan, 1977). The ventral sub-region bi-directionally connects to nuclei in the medial prefrontal cortex, amygdala, and nucleus accumbens either directly from ventral CA1 or routed through the ventral subiculum (Cenquizca and Swanson, 2007), and contributes to the regulation of emotionality and fear (Adhikari et al, 2010; Bannerman et al, 2004; Felix-Ortiz et al, 2013; Kheirbek et al, 2013; Kjelstrup et al, 2002). VH function is also implicated in the encoding (Ambrogi Lorenzini et al, 1997; Goshen et al, 2011; Maren and Holt, 2004; Yoon and Otto, 2007), extinction (Hobin et al, 2006; Sierra-Mercado et al, 2011), and 
generalization (Cullen et al, 2015; McHugh et al, 2013; Yuan et al, 2015) of learned fear responses. Conversely, the dorsal hippocampus receives inputs from regions that are linked to the processing of spatial information (Cenquizca and Swanson, 2007), contributes to spatial cognition (Morris et al, 1982; 1990), and acts as a comparator when faced with ambiguous spatial information (Bannerman et al, 2012; Frankland et al, 1998; Tsien et al, 1996). Heterosynaptic gating of synaptic plasticity in the dorsal hippocampus by the entorhinal cortex may be the circuit-level mechanism supporting discrimination between similar spatial contexts (Basu et al, 2016). Previous work from our laboratory has shown that offspring of high LG dams display a greater capacity for learning and memory in the Morris water maze, a spatial learning test (Bredy et al, 2004; Liu et al, 2000), which in turn associates with an increased capacity for LTP in the dorsal hippocampus (Bagot et al, 2009; Champagne et al, 2008; Nguyen et al, 2015). Intriguingly, despite their clear differences in extrinsic connectivity, functional contribution, and neurophysiology, the internal trisynaptic circuit organization of the hippocampus stays effectively constant throughout the dorso-ventral axis. Therefore, a common computational process may be effected by each sub-region. Each region may instead differ primarily in the type of information it handles, as dictated by the circuit each sub-region functions in (Bannerman et al, 2014). If the ventral hippocampus performs a similar computation to that of the dorsal hippocampus on distinct information modalities defined by its connectivity, this region may contribute to discrimination of learned fear-related cues. Since the ventral hippocampus participates in the formation of nonspatial cued fear, such as auditory cue learning (Maren and Holt, 2004; Yoon and Otto, 2007), we hypothesized that NMDA receptor-dependent $\mathrm{VH}$ plasticity contributes to discrimination of learned auditory cues. We reasoned that increased fearfulness in offspring of low LG dams (Caldji et al, 1998; Menard et al, 2004; Weaver et al, 2006) suggests that neural function in fear-related structures, such as the ventral hippocampus, is similarly increased in these animals.

Although inhibition or ablation of the ventral hippocampus impairs the acquisition and extinction of conditioned cued fear (Maren et al, 1997; Maren and Holt, 2004; Richmond et al, 1999), the formation of direct tone-shock pairings are likely supported by the amygdala (Nabavi et al, 2014; Phillips and LeDoux, 1992). Through its circuit connections with the medial prefrontal cortex and amygdala, the ventral hippocampus is ideally situated to gate amygdaladriven fear signaling. VH CA1 efferents gate neuronal firing and heterosynaptic plasticity in the amygdala through inputs onto inhibitory interneurons (Bazelot et al, 2015; Hubner et al, 2014). Such gating of synaptic plasticity in the dorsal hippocampus by the entorhinal cortex has been linked to generalization of dorsal hippocampal-mediated contextual fear conditioning (Basu et al, 2016), suggesting that this may be a common mechanism critical in the specification of associative memory. Although increased intra-amygdalar inhibition may drive reduced generalization in offspring of low LG mothers (Bergado-Acosta et al, 2008), a previous study examining benzodiazepine binding in the amygdala (which is mediated by $\mathrm{GABA}_{\mathrm{A}}$ receptors) found the opposite effect of maternal care such that receptor binding (ie, expression) was significantly greater in offspring of high LG mothers (Caldji et al, 1998). This rules out the possibility that intra-amygdalar inhibition explains reduced generalization in offspring of low LG mothers, and suggests instead that VH network effects are sufficient to reverse inhibitory deficits in the amygdala and increase memory specificity. The ventral hippocampus may also indirectly gate amygdalar activity by recruiting medial prefrontal cortical nodes into memory traces. The formation of synaptic plasticity in the ventral hippocampus increases theta-slow gamma phase coupling with the medial prefrontal cortex (Zheng and Zhang, 2015), which in turn signals safety following fear learning through inhibition of the amygdala (Likhtik et al, 2014).

As offspring of low LG mothers express larger magnitude of LTP in the ventral hippocampus when compared with offspring of high LG mothers, we investigated the role of $\mathrm{VH}$ LTP during acquisition of tone-shock associations in the expression of generalized fear to neutral cues. Using an interfering peptide approach, we occluded C-terminal phosphorylation of GluA1 to block AMPA receptor insertion into postsynaptic membranes, which is a primary mechanism in the potentiation of glutamatergic synapses (Barria et al, 1997a; 1997b; Rumpel et al, 2005; Shi et al, 2001; Yu et al, 2008). Such plasticity of synaptic function is thought to underlie information storage in neural networks, and is the major biological substrate likely supporting learning and the formation of memories (Bliss and Collingridge, 1993). Inhibition of LTP formation in the ventral hippocampus increased freezing in response to the neutral cue in offspring of low LG mothers, suggesting that VH LTP during fear memory acquisition contributes to the specification of learned fear and prevents later expression of non-specific fear responses. Offspring of high LG mothers display fearassociated freezing in response to the neutral cue that is unaffected by occlusion of VH LTP, which may reflect a ceiling effect resulting from a reduced capacity for VH LTP. In the present studies, we used a moderate current in delivering the unconditioned stimulus. Generalization of conditioned fear increases as a function of stress (Kaouane et al, 2012); thus, at lower shock intensities, a VH LTPdependent discrimination mechanism in offspring of high LG mothers may be unmasked, whereas discrimination may be reduced in offspring of low LG mothers at higher shock intensities. At the shock intensity used in the present studies, however, unconditioned responses were consistent between offspring of high and low LG dams, suggesting that differential sensitivity to shock is not likely to account for the observed differences in memory. Offspring of low LG mothers were also previously shown to be deficient in spatial learning and impaired in dorsal hippocampal LTP (Champagne et al, 2008; Liu et al, 2000), which could affect the acquisition of contextual information in a fear conditioning paradigm. Although deficits in contextual discrimination may underlie the generalization of fear memories (Kaouane et al, 2012), our paradigm differentiates the learning context from the recall context through multiple sensory modalities (tactile, olfactory, and visual) using both proximal and distal cues, therefore minimizing similarities between the conditioning and the retention context. Furthermore, exposure to the differentiated recall context prior to tone presentation did not elicit fear responses in any 
group, indicating that learned and generalized responses were cue-specific.

Although LTP is most often manipulated by targeting its induction mechanism, such as the activation of NMDA receptors, we have previously shown that in the dorsal hippocampus, NMDA receptor blockade differentially suppresses or unmasks dentate gyrus LTP in slices from offspring of high and low LG dams, respectively (Bagot et al, 2012). Thus, we instead used Tat-GluAlct blockade of LTP to inhibit trafficking of AMPA receptors, an expression mechanism for LTP in the hippocampus and other brain regions (Barria et al, 1997a; Barria et al, 1997b; Nicoll, 2003; Rumpel et al, 2005; Shi et al, 2001; Yu et al, 2008). Although the effect of the peptide was validated in uncharacterized animals by recording whole-cell LTP while loading VH CA1 neurons with the decoy or control peptide, we surmised that Tat-GluA1ct infusion similarly but putatively blocks LTP formation in offspring of high and low LG offspring. Future in vivo experiments should explore if changes in synaptic function in the $\mathrm{VH}$ are causally linked to fear generalization. We also note that pharmacological inhibition of AMPA receptor insertion was directed at the ventral hippocampus and employed an injection volume for which the spread is expected to extensively cover the $\mathrm{VH}$ formation (Peterson, 1998). As the attached Tat domain that enables peptide entry into cells is not cell-type specific, AMPA receptor insertion is expected to be blocked ubiquitously within the target region. Whether the reduction in generalization observed in offspring of low LG dams is supported by overall hyperfunction of the trisynaptic circuit and thus augmentation of information processing by the $\mathrm{VH}$ node in fear-processing neural circuits or whether effects are mediated more specifically through programming of a hippocampal subfield (eg, CA1) could be parsed in future studies using cell type-specific manipulations.

Successful identification of threat-associated signals in complex contexts increases survival by forming predictive memories that can inform future encounters. The hippocampal-amygdalar-prefrontal cortical neural circuits that support fear conditioning have been studied extensively, but in studies largely focused on the permissive mechanisms that associate signal-threat pairings. Preventing the inappropriate elicitation of fear responses to neutral stimuli during recall may involve activity in the ventral hippocampus and medial prefrontal cortex (Likhtik et al, 2014; McHugh et al, 2013). Impairment of memory accuracy (eg, by a severe trauma) can reduce the specificity of fear associations, leading to the inability to restrict fear responses to salient contexts and inappropriate generalization of conditioned fear to neutral but salient stimuli (Kaouane et al, 2012). Such maladaptive responses elicited by non-predictive cues must be considered in contrast to the adaptive acquisition and retention of predictive fear memories. This qualitative distinction is critical for pathophysiological implications: the inappropriate elicitation of fear responses in safe contexts by neutral cues may emerge as a result of the maladaptive/pathological nature of the underlying memory, as such generalization characterizes affective disorders such as anxiety and posttraumatic stress (Elzinga and Bremner, 2002). Generalization of conditioned fear in animal models is proposed to more closely reflect the clinical phenomenon of post-traumatic stress, as these paradigms can simultaneously recapitulate

peritraumatic amnesia and hypermnesia for the traumatic stimulus (Layton and Krikorian, 2002). Moreover, exposure to a stressful or fearful learning event does not always lead to fear-related pathology. Inter-individual variability in responses to stress may account for the variable elicitation of pathological fear (Desmedt et al, 2015). We identify two subpopulations of animals that are equally able to acquire the fear memory, but reveal a qualitative difference in the underlying memory formed (ie, offspring of high LG mothers form a generalized memory; offspring of low LG mothers form a specific memory). Thus, maternal care in early life may significantly contribute to the quality of fear memories formed in adulthood, with potential consequences for vulnerability to psychopathology.

Variations in maternal care appear to act as predictive signals for the offspring that shape neurodevelopment in a manner that is consistent with the prevailing environmental conditions. Chronic stress during pregnancy reduces LG in dams (Champagne et al, 2003), producing offspring phenotypes that emphasize fear-related signaling and behaviors (Bagot et al, 2009; Caldji et al, 1998; Liu et al, 1997; Nguyen et al, 2015; Weaver et al, 2006). Offspring of low LG mothers display increased fearfulness and greater HPA responses to stress (Caldji et al, 1998; Liu et al, 1997; Weaver et al, 2005), but simultaneously show greater memory retention when learning under stressful conditions (Bagot et al, 2009; Champagne et al, 2008). In contrast, increased maternal LG associates with more modest HPA responses to stress (Liu et al, 1997), but associates with reduced spatial learning and plasticity under stressful conditions (Bagot et al, 2012; 2009; Champagne et al, 2008). Our present findings support the role for low LG rearing in elaborating signaling and plasticity in fear-related networks, increasing memory accuracy when encoding and recalling fear-related information. This phenotype may prove adaptive in the high-threat environments that associate with low LG rearing (Champagne et al, 2003), facilitating successful identification and discrimination of threat-predictive stimuli. By comparison, offspring of high LG mothers display generalized freezing responses to neutral stimuli following fear learning, suggesting a reduction in the specificity of associative memories containing emotional or fearful information. Such generalization of autonomic fear responses to peritraumatic stimuli may reflect vulnerability for affective system dysregulation following perturbation by a severe stressor. Our findings suggest that the maternal care of low LG mothers can elaborate neural function in a regionspecific manner, emphasizing fear-related neural systems to support greater specificity of fear associations.

Glucocorticoid signaling modulates hippocampal synaptic plasticity in opposite directions in the dorsal and ventral subregion. In rats, NMDA receptor-dependent LTP in the dorsal hippocampus is inhibited by corticosterone acting through glucocorticoid receptors (Krugers et al, 2005; Wiegert et al, 2005), whereas LTP formation in the ventral hippocampus is facilitated by corticosterone acting through a predominantly mineralocorticoid receptor-mediated mechanism (Maggio and Segal, 2007). This differential modulation of dorsal/ ventral plasticity suggests a functional re-routing of hippocampal information during stress events to support fearrelated signals flowing through ventral hippocampusassociated networks (Maggio and Segal, 2012). In the dorsal 
hippocampus, glucocorticoid receptor expression is reduced in offspring of low LG dams, reducing HPA-axis negative feedback sensitivity (Liu et al, 1997; Weaver et al, 2004). A similar mechanism related to differential glucocorticoid receptor expression in the ventral hippocampus may contribute to increased engagement of $\mathrm{VH}$-associated networks in these animals.

In combination with previous findings, these results describe a phenotype where specific fear memories, combined with increased threat monitoring and rapid engagement of threat-response behaviors such as defensive motor programs, are expected to increase an individual's fitness in a highly adverse environment (Bagot et al, 2009; Caldji et al, 1998; Liu et al, 1997; Nguyen et al, 2015; Menard et al, 2004; Weaver et al, 2006). We propose that notions of universally protective or beneficial effects in offspring deriving from dams that display greater frequencies of maternal behaviors are incomplete, failing to account for task-specific advantages that distinct forms of cognition may provide. We propose instead that these effects are better understood from a developmental-evolutionary perspective, in which traits deriving from rearing in an adverse environment confer greater fitness to said adverse environment. In offspring of low LG dams, such hyperfunction of fear signaling arising from adverse rearing conditions may prove adaptive in threatening environments by increasing the capacity of learning systems to sustain accurate informational encoding under high stress, but in excess may contribute to the formation of fear-related psychopathology such as generalized anxiety and major depression.

\section{FUNDING AND DISCLOSURE}

This research was supported by grants from the Natural Sciences and Engineering Research Council of Canada (38868-03) and the Sackler Program for Epigenetics \& Psychobiology at McGill to MJM, and from the Fonds de Recherche du Québec - Santé (31888) to HBN. The authors declare no conflict of interest.

\section{ACKNOWLEDGMENTS}

We thank Dr Chelsea Cavanagh and Dr Joëlle Lopez for assistance with fear conditioning and drug infusions.

\section{REFERENCES}

Adhikari A, Topiwala MA, Gordon JA (2010). Synchronized activity between the ventral hippocampus and the medial prefrontal cortex during anxiety. Neuron 65: 257-269.

Ambrogi Lorenzini CG, Baldi E, Bucherelli C, Sacchetti B, Tassoni G (1997). Role of ventral hippocampus in acquisition, consolidation and retrieval of rat's passive avoidance response memory trace. Brain Res 768: 242-248.

Bagot RC, Parise EM, Pena CJ, Zhang HX, Maze I, Chaudhury D et al (2015). Ventral hippocampal afferents to the nucleus accumbens regulate susceptibility to depression. Nat Commun 6: 7062.

Bagot RC, Tse YC, Nguyen HB, Wong AS, Meaney MJ, Wong TP (2012). Maternal care influences hippocampal N-methyl-Daspartate receptor function and dynamic regulation by corticosterone in adulthood. Biol Psychiatry 72: 491-498.
Bagot RC, van Hasselt FN, Champagne DL, Meaney MJ, Krugers HJ, Joels M (2009). Maternal care determines rapid effects of stress mediators on synaptic plasticity in adult rat hippocampal dentate gyrus. Neurobiol Learn Mem 92: 292-300.

Bannerman DM, Bus T, Taylor A, Sanderson DJ, Schwarz I, Jensen $\mathrm{V}$ et al (2012). Dissecting spatial knowledge from spatial choice by hippocampal NMDA receptor deletion. Nat Neurosci 15: 1153-1159.

Bannerman DM, Rawlins JN, McHugh SB, Deacon RM, Yee BK, Bast $\mathrm{T}$ et al (2004). Regional dissociations within the hippocampus-memory and anxiety. Neurosci Biobehav Rev 28: 273-283.

Bannerman DM, Sprengel R, Sanderson DJ, McHugh SB, Rawlins JN, Monyer $\mathrm{H}$ et al (2014). Hippocampal synaptic plasticity, spatial memory and anxiety. Nat Rev Neurosci 15: 181-192.

Barria A, Derkach V, Soderling T (1997a). Identification of the $\mathrm{Ca} 2+/$ calmodulin-dependent protein kinase II regulatory phosphorylation site in the alpha-amino-3-hydroxyl-5-methyl-4isoxazole-propionate-type glutamate receptor. J Biol Chem 272: 32727-32730.

Barria A, Muller D, Derkach V, Griffith LC, Soderling TR (1997b). Regulatory phosphorylation of AMPA-type glutamate receptors by CaM-KII during long-term potentiation. Science 276: 2042-2045.

Basu J, Zaremba JD, Cheung SK, Hitti FL, Zemelman BV, Losonczy A et al (2016). Gating of hippocampal activity, plasticity, and memory by entorhinal cortex long-range inhibition. Science 351: aaa5694.

Bazelot M, Bocchio M, Kasugai Y, Fischer D, Dodson PD, Ferraguti F et al (2015). Hippocampal theta input to the amygdala shapes feedforward inhibition to gate heterosynaptic plasticity. Neuron 87: 1290-1303.

Bergado-Acosta JR, Sangha S, Narayanan RT, Obata K, Pape HC, Stork O (2008). Critical role of the $65-\mathrm{kDa}$ isoform of glutamic acid decarboxylase in consolidation and generalization of Pavlovian fear memory. Learn Mem 15: 163-171.

Bittner KC, Andrasfalvy BK, Magee JC (2012). Ion channel gradients in the apical tuft region of CA1 pyramidal neurons. PloS ONE 7: e46652.

Bliss TV, Collingridge GL (1993). A synaptic model of memory: long-term potentiation in the hippocampus. Nature 361: 31-39.

Bredy TW, Zhang TY, Grant RJ, Diorio J, Meaney MJ (2004). Peripubertal environmental enrichment reverses the effects of maternal care on hippocampal development and glutamate receptor subunit expression. Eur J Neurosci 20: 1355-1362.

Caldji C, Tannenbaum B, Sharma S, Francis D, Plotsky PM, Meaney MJ (1998). Maternal care during infancy regulates the development of neural systems mediating the expression of fearfulness in the rat. Proc Natl Acad Sci USA 95: 5335-5340.

Calfa G, Bussolino D, Molina VA (2007). Involvement of the lateral septum and the ventral Hippocampus in the emotional sequelae induced by social defeat: role of glucocorticoid receptors. Behav Brain Res 181: 23-34.

Cenquizca LA, Swanson LW (2007). Spatial organization of direct hippocampal field CA1 axonal projections to the rest of the cerebral cortex. Brain Res Rev 56: 1-26.

Champagne DL, Bagot RC, van Hasselt F, Ramakers G, Meaney MJ, de Kloet ER et al (2008). Maternal care and hippocampal plasticity: evidence for experience-dependent structural plasticity, altered synaptic functioning, and differential responsiveness to glucocorticoids and stress. J Neurosci 28: 6037-6045.

Champagne FA, Francis DD, Mar A, Meaney MJ (2003). Variations in maternal care in the rat as a mediating influence for the effects of environment on development. Physiol Behav 79: 359-371.

Ciocchi S, Passecker J, Malagon-Vina H, Mikus N, Klausberger T (2015). Brain computation. Selective information routing by ventral hippocampal CA1 projection neurons. Science 348: 560-563.

Cullen PK, Gilman TL, Winiecki P, Riccio DC, Jasnow AM (2015). Activity of the anterior cingulate cortex and ventral hippocampus 
underlie increases in contextual fear generalization. Neurobiol Learn Mem 124: 19-27.

Desmedt A, Marighetto A, Piazza PV (2015). Abnormal fear memory as a model for posttraumatic stress disorder. Biol Psychiatry 78: 290-297.

Dougherty KA, Islam T, Johnston D (2012). Intrinsic excitability of CA1 pyramidal neurones from the rat dorsal and ventral hippocampus. J Physiol 590: 5707-5722.

Elzinga BM, Bremner JD (2002). Are the neural substrates of memory the final common pathway in posttraumatic stress disorder (PTSD)? J Affect Disord 70: 1-17.

Felix-Ortiz AC, Beyeler A, Seo C, Leppla CA, Wildes CP, Tye KM (2013). BLA to vHPC inputs modulate anxiety-related behaviors. Neuron 79: 658-664.

Frankland PW, Cestari V, Filipkowski RK, McDonald RJ, Silva AJ (1998). The dorsal hippocampus is essential for context discrimination but not for contextual conditioning. Behav Neurosci 112: 863-874.

Gee DG, Gabard-Durnam LJ, Flannery J, Goff B, Humphreys KL, Telzer EH et al (2013). Early developmental emergence of human amygdala-prefrontal connectivity after maternal deprivation. Proc Natl Acad Sci USA 110: 15638-15643.

Goshen I, Brodsky M, Prakash R, Wallace J, Gradinaru V, Ramakrishnan C et al (2011). Dynamics of retrieval strategies for remote memories. Cell 147: 678-689.

Hobin JA, Ji J, Maren S (2006). Ventral hippocampal muscimol disrupts context-specific fear memory retrieval after extinction in rats. Hippocampus 16: 174-182.

Hubner C, Bosch D, Gall A, Luthi A, Ehrlich I (2014). Ex vivo dissection of optogenetically activated mPFC and hippocampal inputs to neurons in the basolateral amygdala: implications for fear and emotional memory. Front Neurosci 8: 64.

Jin J, Maren S (2015). Fear renewal preferentially activates ventral hippocampal neurons projecting to both amygdala and prefrontal cortex in rats. Sci Rep 5: 8388.

Kaouane N, Porte Y, Vallee M, Brayda-Bruno L, Mons N, Calandreau L et al (2012). Glucocorticoids can induce PTSDlike memory impairments in mice. Science 335: 1510-1513.

Kheirbek MA, Drew LJ, Burghardt NS, Costantini DO, Tannenholz L, Ahmari SE et al (2013). Differential control of learning and anxiety along the dorsoventral axis of the dentate gyrus. Neuron 77: 955-968.

Kjelstrup KG, Tuvnes FA, Steffenach HA, Murison R, Moser EI, Moser MB (2002). Reduced fear expression after lesions of the ventral hippocampus. Proc Natl Acad Sci USA 99: $10825-10830$.

Krugers HJ, Alfarez DN, Karst H, Parashkouhi K, van Gemert N, Joels M (2005). Corticosterone shifts different forms of synaptic potentiation in opposite directions. Hippocampus 15: 697-703.

Layton B, Krikorian R (2002). Memory mechanisms in posttraumatic stress disorder. J Neuropsychiatry Clin Neurosci 14: 254-261.

Likhtik E, Stujenske JM, Topiwala MA, Harris AZ, Gordon JA (2014). Prefrontal entrainment of amygdala activity signals safety in learned fear and innate anxiety. Nat Neurosci 17: 106-113.

Liu D, Diorio J, Day JC, Francis DD, Meaney MJ (2000). Maternal care, hippocampal synaptogenesis and cognitive development in rats. Nat Neurosci 3: 799-806.

Liu D, Diorio J, Tannenbaum B, Caldji C, Francis D, Freedman A et al (1997). Maternal care, hippocampal glucocorticoid receptors, and hypothalamic-pituitary-adrenal responses to stress. Science 277: 1659-1662.

Maggio N, Segal M (2007). Striking variations in corticosteroid modulation of long-term potentiation along the septotemporal axis of the hippocampus. J Neurosci 27: 5757-5765.

Maggio N, Segal M (2012). Steroid modulation of hippocampal plasticity: switching between cognitive and emotional memories. Front Cell Neurosci 6: 12.
Maren S, Aharonov G, Fanselow MS (1997). Neurotoxic lesions of the dorsal hippocampus and Pavlovian fear conditioning in rats. Behav Brain Res 88: 261-274.

Maren S, Holt WG (2004). Hippocampus and Pavlovian fear conditioning in rats: muscimol infusions into the ventral, but not dorsal, hippocampus impair the acquisition of conditional freezing to an auditory conditional stimulus. Behav Neurosci 118: 97-110.

McHugh SB, Marques-Smith A, Li J, Rawlins JN, Lowry J, Conway M et al (2013). Hemodynamic responses in amygdala and hippocampus distinguish between aversive and neutral cues during Pavlovian fear conditioning in behaving rats. Eur J Neurosci 37: 498-507.

Menard JL, Champagne DL, Meaney MJ (2004). Variations of maternal care differentially influence 'fear' reactivity and regional patterns of cFos immunoreactivity in response to the shock-probe burying test. Neuroscience 129: 297-308.

Morris RG, Garrud P, Rawlins JN, O'Keefe J (1982). Place navigation impaired in rats with hippocampal lesions. Nature 297: 681-683.

Morris RG, Schenk F, Tweedie F, Jarrard LE (1990). Ibotenate lesions of hippocampus and/or subiculum: dissociating components of allocentric spatial learning. Eur J Neurosci 2: 1016-1028.

Nabavi S, Fox R, Proulx CD, Lin JY, Tsien RY, Malinow R (2014). Engineering a memory with LTD and LTP. Nature 511: 348-352.

Nguyen HB, Bagot RC, Diorio J, Wong TP, Meaney MJ (2015). Maternal care differentially affects neuronal excitability and synaptic plasticity in the dorsal and ventral hippocampus. Neuropsychopharmacology 40: 1590-1599.

Nicoll RA (2003). Expression mechanisms underlying long-term potentiation: a postsynaptic view. Philos Trans $R$ Soc Lond B Biol Sci 358: 721-726.

Peterson SL (1998). Drug microinjection in discrete brain regions. Kopf Carrier 50: 1-6.

Phillips RG, LeDoux JE (1992). Differential contribution of amygdala and hippocampus to cued and contextual fear conditioning. Behav Neurosci 106: 274-285.

Pollak SD, Kistler DJ (2002). Early experience is associated with the development of categorical representations for facial expressions of emotion. Proc Natl Acad Sci USA 99: 9072-9076.

Richmond MA, Yee BK, Pouzet B, Veenman L, Rawlins JN, Feldon J et al (1999). Dissociating context and space within the hippocampus: effects of complete, dorsal, and ventral excitotoxic hippocampal lesions on conditioned freezing and spatial learning. Behav Neurosci 113: 1189-1203.

Risold PY, Swanson LW (1996). Structural evidence for functional domains in the rat hippocampus. Science 272: 1484-1486.

Rumpel S, LeDoux J, Zador A, Malinow R (2005). Postsynaptic receptor trafficking underlying a form of associative learning. Science 308: 83-88.

Shi S, Hayashi Y, Esteban JA, Malinow R (2001). Subunit-specific rules governing AMPA receptor trafficking to synapses in hippocampal pyramidal neurons. Cell 105: 331-343.

Sierra-Mercado D, Padilla-Coreano N, Quirk GJ (2011). Dissociable roles of prelimbic and infralimbic cortices, ventral hippocampus, and basolateral amygdala in the expression and extinction of conditioned fear. Neuropsychopharmacology 36: 529-538.

Swanson LW, Cowan WM (1977). An autoradiographic study of the organization of the efferent connections of the hippocampal formation in the rat. J Compar Neurol 172: 49-84.

Tsien JZ, Huerta PT, Tonegawa S (1996). The essential role of hippocampal CA1 NMDA receptor-dependent synaptic plasticity in spatial memory. Cell 87: 1327-1338.

Weaver IC, Cervoni N, Champagne FA, D'Alessio AC, Sharma S, Seckl JR et al (2004). Epigenetic programming by maternal behavior. Nat Neurosci 7: 847-854.

Weaver IC, Champagne FA, Brown SE, Dymov S, Sharma S, Meaney MJ et al (2005). Reversal of maternal programming of stress responses in adult offspring through methyl 
supplementation: altering epigenetic marking later in life. $J$ Neurosci 25: 11045-11054.

Weaver IC, Meaney MJ, Szyf M (2006). Maternal care effects on the hippocampal transcriptome and anxiety-mediated behaviors in the offspring that are reversible in adulthood. Proc Natl Acad Sci USA 103: 3480-3485.

Weeden CS, Hu NJ, Ho LU, Kesner RP (2014). The role of the ventral dentate gyrus in olfactory pattern separation. Hippocampus 24: 553-559.

Wiegert O, Pu Z, Shor S, Joels M, Krugers H (2005). Glucocorticoid receptor activation selectively hampers $\mathrm{N}$-methyl-D-aspartate receptor dependent hippocampal synaptic plasticity in vitro. Neuroscience 135: 403-411.
Yoon T, Otto T (2007). Differential contributions of dorsal vs. ventral hippocampus to auditory trace fear conditioning. Neurobiol Learn Mem 87: 464-475.

Yu SY, Wu DC, Liu L, Ge Y, Wang YT (2008). Role of AMPA receptor trafficking in NMDA receptor-dependent synaptic plasticity in the rat lateral amygdala. J Neurochem 106: 889-899.

Yuan RK, Hebert JC, Thomas AS, Wann EG, Muzzio IA (2015). HDAC I inhibition in the dorsal and ventral hippocampus differentially modulates predator-odor fear learning and generalization. Front Neurosci 9: 319.

Zheng C, Zhang T (2015). Synaptic plasticity-related neural oscillations on hippocampus-prefrontal cortex pathway in depression. Neuroscience 292: 170-180.

Supplementary Information accompanies the paper on the Neuropsychopharmacology website (http://www.nature.com/npp) 